\title{
RESEARCH ON THE MAPPING OF LARGE-SCALE TOPOGRAPHIC MAPS BASED ON LOW-ALTITUDE DRONE AERIAL PHOTOGRAPHY SYSTEM
}

\author{
Rongshan $\mathrm{Lu}^{1, *}$ \\ ${ }^{1}$ Bowen College of Management GuiLin University of Technology, GuiLin, China
}

KEY WORDS: UAV aerial survey system, Digital Orthophoto Map (DOM), Digital Elevation Model (DEM), GPS

\begin{abstract}
:
The field data collection and in-house data processing of the aerial photography system of the uavouring aircraft are studied, combined with their working examples in this paper. The aerial survey of the Bowen School of Management of Guilin University of Technology is carried out by using the Southern Sky Patrol AS1200 aerial survey system, and the obtained data is processed and studied in the internal and external industry. In order to successfully complete this research, this article (1) first of all to the external industry collected data inspection, that is, the quality of the aircraft film inspection, the main content of the inspection is the detection of the image of the tone, resolution, level, whether the film can clearly identify the main objects, such as houses, roads, etc. can be clearly identified; - Whether the film is ghosting, whether there is misalignment and fuzziness, etc. , if the film has blurred or other quality problems, it is necessary to carry out retest or retest, this step is mainly to ensure the quality of research data, for the industry processing data to do a basic guarantee. (2) For the processing of in-house data, this paper uses a correction model for image data distortion pre-processing, the method of arithmetic and the edge detection operator method of extracting the image feature, and the image matching method Greenfeld-Schenk method based on the feature, Bamard-Thompson method and jump method and other methods to match the image, at the same time, this paper also takes the aerial triangulation method of the processing of internal data, complete the measurement control point encryption work, so as to calculate the spatial coordinates of the encryption control point, reduce the field laying work like control points, and thus reduce the working time of the field. (3) For improving the accuracy of the topographic elevation point in the aviation area, this paper uses polymorphic curve to be legal, using the field elevation measured value of the checkpoint and the three-dimensional measurement value does not match the difference, using the least square-bymultiply method to fit, so that the elevation checkpoint curve fitting value change and the actual difference is the smallest, with the measurement area elevation accuracy.
\end{abstract}

\section{INTRODUCTION}

In the 1970 s, due to the emergence of electronic speedometers, large-scale digital mapping technology began to take off. In the early 1980s, the advent of full-station electronic speedometers became more common in the study of digital mapping technology (Gao et al., 2015). At present, the more common domestic and foreign station instruments, such as: Leica, Tianbao, Topkang, etc. have started at this time, the use of the whole station instrument mapping large-scale digital topographic map methods mainly include: sketching method, electronic flat method, object attribute coding method and measurement method. The advantages of using GPS technology and full station instrument to carry out 1:500 large scale rural land survey are very obvious, and the combined application of the two makes the accuracy of the map being guaranteed (Yang, 2015). RTK technology is applied to large scale scale maps, which makes the technical method of large scale scale mapping more convenient, and also improves the quality of the results. In-vehicle measurement system has been well used in the collection of data, high-density point cloud computing, and mapping intelligence in land tenure work (Zhang et al., 2016).

Modern drones are popular in related industries for their unique superiority and uniqueness, especially in the wide range of areas where drone photogrammetry technology serves (Herwitz et al., 2002; Herwitz et al., 2004; Remondino et al., 2011. In the production of large-scale topographic maps of UAV photogrammetry systems, for the uavouring image processing system, the mainstream software abroad has the Swiss pix4D production PIX4DMAPPER drone data and aerial image processing software, Europe Inpho's Inpho Photogrammetry System and the "Pixel Factory" developed by Info Terra (Ma, 2018). China mainly has Zhang Zuxun academician developed the grid processing system DPGrid system and JX-4 system, Beijing Tsinghua Shanwei's EPS digital 3D mapping software and space vision company's new digital photogrammetry system Map Matrix. Zheng Huiqin discusses the key technology of the 1:1000 scale scale map based on the Wuyi mountain measuring area based on the aerial image of the drone and the feasibility of its production (Deng, 2011). Shen Jie et al. (2017) obtained image data using small drones, and verified the advantages of low-altitude photogrammetry of drones in the design and application of water conservancy facilities.

The paper discusses the process of obtaining the external image data of the aerial photography system of the drone and the processing of the in-house image data, and describes the method of producing digital topographic maps based on the production of aerial photography data of the drone. Through the processing of data obtained from aerial survey of drones, the production of digital topographic maps and precision analysis provide scant new ideas for the production of digital topographic maps, and lay a theoretical foundation for practical application.

\footnotetext{
* Corresponding author

41156203@qq.com
} 


\section{EXPERIMENTAL DATA EXPERIMENTAL DATA AND ITS PROCESSING}

\subsection{Experimental data acquisition}

This aerial survey is based on the experiment of Yanshan Campus of Bowen School of Management, Guilin University of Technology. The campus is located in Yanshan District, Guilin City. It is located in the northeast of Guangxi Zhuang Autonomous Region, south of Guilin City. It was established at the end of 1996. It is connected to Lingchuan County in the east, Lingui District in the west, Yangshuo County in the south, and Xiangshan in the north. District and Qixing District. Yanshan District is a typical karst area, with multiple rocky mountains and hilly landforms. It is located in a low latitude zone and belongs to the subtropical monsoon climate. Guilin University of Technology Bowen School of Management is located in the southwest of Yanshan Town, Guilin City, about 45 kilometers from the city center. It is connected to Guilin University of Technology and covers an area of about 800 acres. The survey area is relatively dense, and the flow of people is particularly large. The degree of terrain fluctuation in the survey area is relatively obvious. The Bowen School of Management of Guilin University of Technology newly purchased a Southern Sky Sight AS1200 drone and an associated image processing system. Now, in order to update the campus geographic information data, and also to test the equipment, the aerial photography operation of the test area is carried out. The study area is shown in Figure 1 as follows:

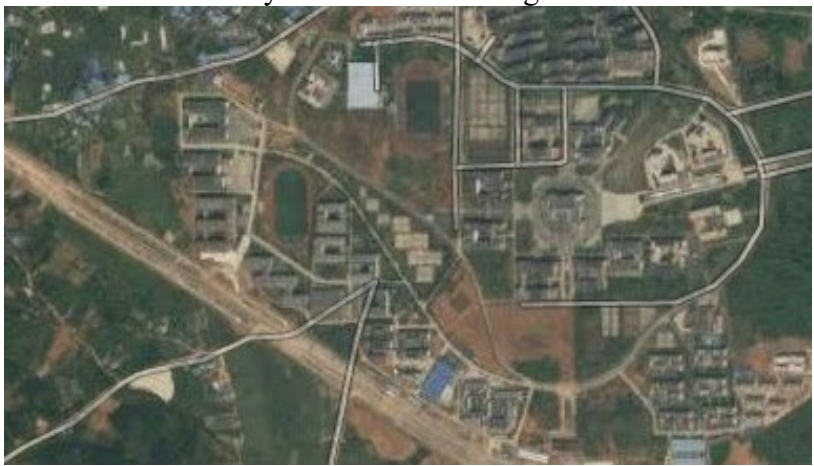

Figure 1. Research Area of the Bowen School of Management, Guilin University of Technology

University of Technology

If the results of the inspection do not meet the accuracy requirements of in-house data processing, retestor or retest of areas with poor image quality. Choose a clear, cloudless weather during the retest to ensure that the tone of the images collected twice remains basically the same.

\subsection{Data preparation and pre-processing}

\section{(a) Data preparation}

Data pre-processing needs to be aerial cameras, camera exposure parameters, POS files and other corresponding data ready to complete. All images must be placed under a unified path, and the image name must be the same as the POS data name. All relevant documents of the camera need to be manually organized according to the camera check file, the camera's files include: camera focal length, like the main point, CCD size, radial distortion coefficient, pixel size, eccentric distortion coefficient, CCD non-orthogonal distortion coefficient and non-PIXEL square scale coefficient, separator using spaces.

(b) Data pre-processing

After the Southern Sky Patrol AS1200 drone aerial photography collected data, in the use of Southern UAV imaging industry data processing before simple collating the data, the recorded POS data file format to make corresponding modifications, because the IMPORTed TO SouthUAV POS data to meet the system's format requirements, Otherwise, you cannot import into the SouthUAV system.

Open the South SouthUAV system, create a new workspace, according to the corresponding work interface projects, in turn select the calibration, flight video, POS, image control point and checkpoint file import video data. Images should be arranged in accordance with the route, the order can not be wrong, there can be no omission, otherwise can not carry out follow-up processing work. POS files and control files need to determine the file type, and checkpoints need to be consistent with the handle file type. The system automatically calculates the parameters of the image, the orientation work is done automatically, this process does not require human intervention. If you are using a Southern drone, POS data can be selected directly by default type, and if POS files or controls are not the default format of Southern drones, the POS format needs to be re-customized and data can be processed after the data is imported.

\section{RESEARCH METHODS}

\subsection{Aerial triangulation}

Aerial triangulation is the key process of image data processing, including the relative orientation of automatic extraction of connection points and absolute orientation by the calculation of the flat difference after the prickle control point. The purpose of relative orientation is to restore the positions of the two pieces that make up the pair, to create the spatial position characteristic vector stakes of the subject, and to obtain the relative orientation parameters for each vector. Like pairs have two forms of continuity and individuality, solid relative orientation is also divided into two according to the form of the image pair. The relative orientation of the continuous image pair takes the left piece as the reference base, deduces the 5 relative directional elements of the right piece relative to the left piece, and then can use the principle of the spatial front intersection, solve the spatial auxiliary coordinates of the model points, and construct the digital model. The stereo model vector created by relative orientation is located in a space-assisted coordinate system with no fixed scale factor, while absolute orientation is the implementation process of transforming image coordinates and camera orientation to spatial geographic coordinate systems (Lu, 2014).

After using the SouthUAV system to enter the empty three solution page, you can select the processing strategy in the middle drop-down box: efficiency priority, precision priority. Execute the start calculation command, the computer will enter the empty three solution process autonomously. In the process of solving the system, according to the degree of overlap and the attitude of the image, some images are appropriately deleted, so the original data must be backed up in other paths before starting the work.

In the process of solving, according to the overlap and image posture, some images will be appropriately deleted, so before starting the job must be the original data backed up under other paths. The output output after the completion of empty three 
encryption is the DOM described in image form and the DSM described in the form of point cloud.

\subsection{Polyamorphic curves are legal}

By establishing a polynomial curve mathematical model, the elevation correction is simulated, and the mismatch between the field elevation measured value and the stereoscopic measurement value of the checkpoint is used to fit the minimum square-by-multiplier method, so that the change of value and actual difference between the curve fitting at the elevation checkpoint is minimal, so as to improve the accuracy of the elevation of the measuring area.

Assuming that the field elevation value of the checkpoint is $Z$, the elevation stereoscopic measurement is $Z^{\prime}$, when the two are not equal, the non-conformity value is $\Delta \mathrm{Z}=\mathrm{Z}-\mathrm{Z}^{\prime}$, the $\Delta \mathrm{Z}$ as the elevation correction value, the second multiple expressions are as follows:

$$
\Delta \mathrm{Z}=\mathrm{a}_{0}+\mathrm{a}_{1} \mathrm{Z}^{\prime}+\mathrm{a}_{2} \mathrm{Z}^{\prime 2}
$$

There are 3 pending parameters in the upper formula, and at least 3 checkpoints are selected from the elevation checkpoint, which is calculated by the column equation. According to the excess observations, the polynomial error equation can be listed, the correction parameters can be calculated using the indirect flat difference method, $Z^{\prime}$ as the observation.

$$
Z-\left(Z^{\prime}+v_{z}\right)=\Delta Z
$$

The error equation is:

$$
-\mathrm{v}_{\mathrm{z}}=\mathrm{a}_{0}+\mathrm{a}_{1} \mathrm{Z}^{\prime}+\mathrm{a}_{2} \mathrm{Z}^{\prime 2}-1_{\mathrm{z}}
$$

Where, $1_{z}=Z-Z^{\prime}$, select $n$ elevation checkpoints, you can list the error equations, and rewrite the error equations into matrix form as follows:

$$
\mathrm{V}=\mathrm{AX}-\mathrm{L}
$$

The parameters to be determined are calculated using the principle of least squares.

$$
\mathrm{X}=\left(\mathrm{A}^{\mathrm{T}} \mathrm{A}\right)^{-1}\left(\mathrm{~A}^{\mathrm{T}} \mathrm{L}\right)
$$

The calculated undetermined parameter value is substituted into the formula, and according to the elevation stereo measurement value $Z^{\prime}$ of the checkpoint, the elevation correction value $\Delta \mathrm{Z}$ of the checkpoint can be calculated, and the corrected elevation value is calculated. The corrected elevation value is compared with the measured value of the field elevation, and then the elevation accuracy index that can be achieved is evaluated.

\subsection{Elevation surface fitting}

Let the elevation correction value be $\xi$, f $(\mathrm{x}, \mathrm{y})$ be the fitting trend surface of $\xi$ with respect to $(\mathrm{x}, \mathrm{y})$, and $\tau$ be the fitting error. The equation is expressed as follows:

$$
\xi=\mathrm{f}(\mathrm{x}, \mathrm{y})+\tau
$$

In the above formula,

$$
f(x, y)=a_{1} x+a_{2} y+a_{3} x y+a_{4} x^{2}
$$

$$
\begin{gathered}
\xi=\left[\begin{array}{l}
\xi_{1} \\
\xi_{2} \\
\xi_{3} \\
\xi_{4}
\end{array}\right], \quad \mathrm{C}=\left[\begin{array}{l}
\mathrm{a}_{1} \\
\mathrm{a}_{2} \\
\mathrm{a}_{3} \\
\mathrm{a}_{4}
\end{array}\right]^{\mathrm{T}}, \quad \tau=\left[\begin{array}{c}
\tau_{1} \\
\tau_{2} \\
\tau_{3} \\
\tau_{4}
\end{array}\right], \\
X=\left[\begin{array}{cccc}
\mathrm{X}_{1} & \mathrm{X}_{2} & \mathrm{X}_{3} & \mathrm{X}_{4} \\
\mathrm{Y}_{1} & \mathrm{Y}_{2} & \mathrm{Y}_{3} & \mathrm{Y}_{4} \\
\mathrm{X}_{1} \mathrm{Y}_{1} & \mathrm{X}_{2} \mathrm{Y}_{2} & \mathrm{X}_{3} \mathrm{Y}_{3} & \mathrm{X}_{4} \mathrm{Y}_{4} \\
\mathrm{X}_{1}^{2} & \mathrm{X}_{2}^{2} & \mathrm{X}_{3}^{2} & \mathrm{X}_{4}^{2}
\end{array}\right]
\end{gathered}
$$

\subsection{Akima fitting method}

Algorithm principle of Akima fitting method: Akima fitting method has clear requirements for fixed point data. It requires mutual interpolation between measured points. The measured values of these two points are measured at four adjacent points. Must be used at the same time. That is, two measured points and four adjacent points, the measured values of a total of six points can be interpolated. Assuming that the known point data is $\left(\mathrm{x}_{\mathrm{i}}, \mathrm{y}_{\mathrm{i}}\right)(\mathrm{i}=1,2,3 \cdots, \mathrm{n})$, it is first necessary to establish a curve function $\mathrm{y}=\mathrm{f}(\mathrm{x})$, which satisfies the function $\zeta_{\mathrm{i}}=\mathrm{f}\left(\mathrm{x}_{\mathrm{i}}\right)$ and $\mathrm{f}(\mathrm{x})$ functions in a first-order continuous guide, and at the same time, uses a cubic polynomial method to interpolate between any two adjacent points.

Suppose there are $\mathrm{n}$ checkpoints for $\mathrm{x}_{0}<\mathrm{x}_{1}<\mathrm{x}_{2}<\cdots<\mathrm{x}_{\mathrm{n}-1}$ and the elevation difference is $\zeta_{\mathrm{i}}(\mathrm{i}=0,1,2, \cdots, \mathrm{n}-1)$. On its subinterval $\left[\mathrm{x}_{\mathrm{k}}, \mathrm{x}_{\mathrm{k}+1}\right](\mathrm{k}=0,1,2, \cdots \mathrm{n}-2)$, if there are four conditions at the two endpoints:

$$
\left\{\begin{array}{c}
\zeta_{k}=f\left(x_{k}\right) \\
\zeta_{k+1}=f\left(x_{k+1}\right) \\
\zeta_{k}^{\prime}=g_{k} \\
\zeta_{k+1}^{\prime}=g_{k+1}
\end{array}\right.
$$

Where $g_{k}$ and $g_{k+1}$ are uniquely determined from the Akima condition, the elevation value at the fitted point can be solved by establishing a cubic polynomial in the $\left[\mathrm{x}_{\mathrm{k}}, \mathrm{x}_{\mathrm{k}+1}\right](\mathrm{k}=0,1,2, \cdots \mathrm{n}-2)$ interval:

$$
\begin{aligned}
& \mathrm{s}(\mathrm{x})=\mathrm{s}_{0}+\mathrm{s}_{1}\left(\mathrm{x}-\mathrm{x}_{\mathrm{k}}\right)+\mathrm{s}_{2}\left(\mathrm{x}-\mathrm{x}_{\mathrm{k}}\right)^{2}+\mathrm{s}_{3}\left(\mathrm{x}-\mathrm{x}_{\mathrm{k}}\right)^{3} \\
& \left\{\begin{array}{c}
\mathrm{s}_{0}=\zeta_{\mathrm{k}} \\
\mathrm{s}_{1}=\mathrm{t}_{\mathrm{k}} \\
\mathrm{s}_{2}=\left[3\left(\zeta_{\mathrm{k}+1}-\zeta_{\mathrm{k}}\right) /\left(\mathrm{x}_{\mathrm{k}+1}-\mathrm{x}_{\mathrm{k}}\right)-2 \mathrm{t}_{\mathrm{k}}-\mathrm{t}_{\mathrm{k}+1}\right] /\left(\mathrm{x}_{\mathrm{k}+1}-\mathrm{x}_{\mathrm{k}}\right) \\
\mathrm{s}_{3}=\left[\mathrm{t}_{\mathrm{k}}+\mathrm{t}_{\mathrm{k}+1}-2\left(\zeta_{\mathrm{k}+1}-\zeta_{\mathrm{k}}\right) /\left(\mathrm{x}_{\mathrm{k}+1}-\mathrm{x}_{\mathrm{k}}\right)\right] /\left(\mathrm{x}_{\mathrm{k}+1}-\mathrm{x}_{\mathrm{k}}\right)^{2}
\end{array}\right.
\end{aligned}
$$

Where $t_{k}$ and $t_{k+1}$ are the slopes of the measured points of $k$ and $\mathrm{k}+1$, the formula is as follows:

$$
t_{i}=\left(\left|m_{i+1}-m_{i}\right| \cdot m_{i-1}+\left|m_{i-1}-m_{i-2}\right| \cdot m_{i}\right) /\left(\left|m_{i+1}-m_{i}\right|+\left|m_{i-1}-m_{i-2}\right|\right)
$$




\section{ANALYSIS OF EXPERIMENTAL RESULTS AND ELEVATION ACCURACY IMPROVEMENT}

\subsection{Digital Orthographic Image (DOM) Production}

A digital orthophoto map (DOM) is a set of orthophoto images that correct and nest the elements of a photographic piece in turn, and trim it to create a set of orthophoto images with a specific standard frame. DOM has the advantages of high accuracy, large amount of information, content enrichment, very fast production, and strong current potential. There are many ways to make DOM, mainly: orthographic correction, differential correction, full digital photogrammetry, etc. The results of the digital orthophoto map (DOM) generated in this article are shown in Figure 2.

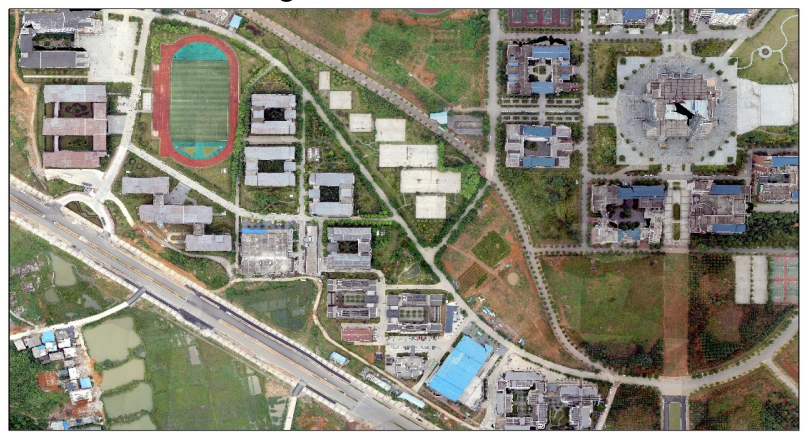

Figure 2(a) Digital orthophoto map (DOM) results

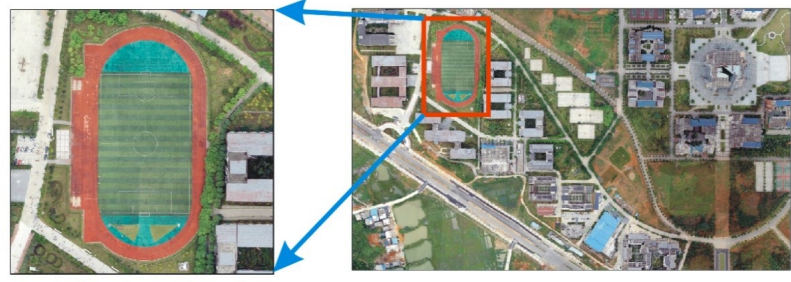

Figure 2(b) Local Digital Orthographic Image (DOM) Results

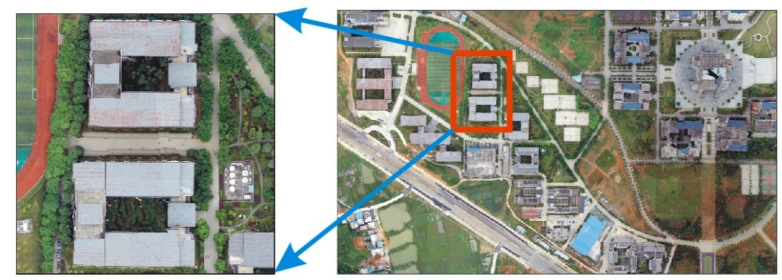

Figure 2(c) Local Digital Orthographic Image (DOM) Results

\subsection{Digital Surface Model (DSM) and Digital Elevation Model (DEM) production}

The Digital Elevation Model (DEM) is a numerical representation of the high and low fluctuations of the surface morphology, presented by a collection of regular points. It is basically consistent with the Digital Surface Model (DSM) when representing areas where the ground is undulating (Fu et al., 2012; He et al., 2012; Li et al., 2012). The key difference between DEM and DSM is that DEM does not include land types such as trees and buildings. It is necessary to edit the DSM before making a DEM. There are many ways to establish DEM: (i) the traditional measurement method is to collect coordinate data directly in the field by total station, GPS, etc.; (ii) to obtain from existing maps; (iii) to use photogrammetric techniques. In this paper, the method of UAV photogrammetry is used to collect the point coordinate data, and the digital orthophoto image is further processed to produce a digital elevation model (DEM). The main workflow is to first generate a point cloud data set based on image matching and absolute orientation, and then further establish a regular DEM according to the high-density point cloud data set.

After the empty three encryption solution is completed, a digital surface model named dsm.ply will be generated in the survey area project directory. The format saved by the Digital Surface Model (DSM) is point cloud data, which can be browsed by point cloud through a browsing tool. The digital surface model is shown in Figure 3.

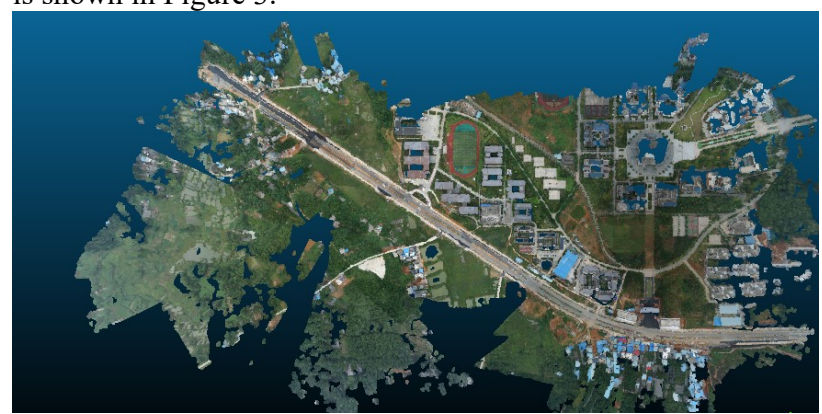

Figure 3 Digital Surface Model (DSM) result After editing the digital surface model (DSM), DEM is generated in the form of a point cloud. Currently, many GIS applications require a rasterized digital elevation model (DEM), so the expression in the form of a point cloud is further converted into Expressed in raster form. Figure 4 below shows the DEM with a pseudo-height elevation picture with a $0.5 \mathrm{~m}$ interval.

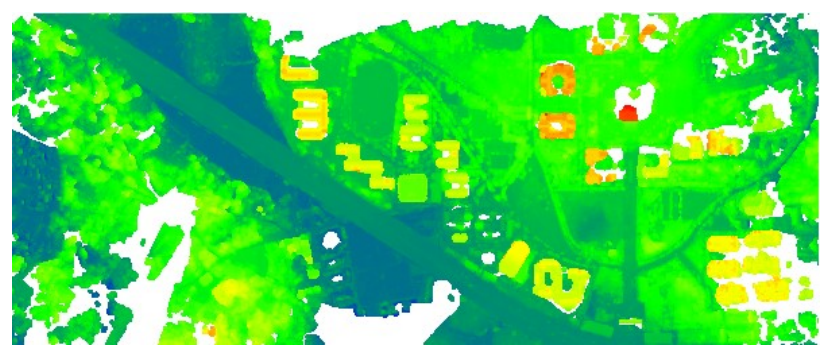

Figure 4 Pseudo-color elevation digital elevation model (DEM) results

\subsection{Digital topographic map production}

DOM has high accuracy, large amount of information and is more intuitive. It has been widely used in urban and regional planning (Chen, 2011; Zhang et al, 2009; Zhang et Al, 2014), it is also possible to produce a digital map with a large scale. At this time, we need to import the empty three results exported by SouthUAV into the digital photogrammetric workstation, collect the terrain feature data, make the digital line graph (DLG), and finally generate the digital topographic map.

For some features that are ambiguous or have shadows, they should be marked immediately for the outside industry to make up for the omission. The field mapping and repairing tests mainly use the total station, GPS and other measurement tools to compile the original picture as the base map, characterize the internal collection, and correct the contents of the collected features, strictly follow the low-altitude The relevant requirements in the Digital Photogrammetry Field Practices guarantee the quality of mapping (Tian, 2014). Finally, the digital line graph (DLG) is edited into the required digital topographic map according to the data format requirements of the Southern CASS mapping software. As shown in Figure 5. 


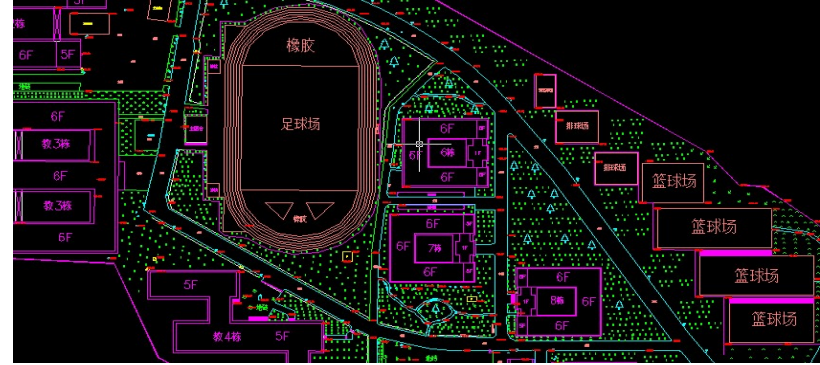

Figure 5 Local topographic map of the survey area 4.4 Improved analysis of elevation accuracy

In the paper, the polynomial curve mathematical model, the elevation surface fitting method and the Akima curve fitting method are used to curve the elevation points of the topographic maps in the aerial survey area. Eight elevation checkpoints with relatively high precision are selected in the work area to solve the polynomial parameters of the elevation correction model, and the remaining 12 points are compared as the accuracy check before and after the elevation fit.

\subsubsection{Correction of elevation by polynomial curve fitting} According to the elevation data of 12 points, the maximum and minimum values of the elevation error of the checkpoint after correction are $0.40 \mathrm{~m}$ and $0.03 \mathrm{~m}$, respectively, and the error in the corrected elevation is also calculated to be $0.240 \mathrm{~m}$. The elevation error distribution before and after the checkpoint correction is as shown in Figure 6.

Comparison of the accuracy of polynomial curve fitting

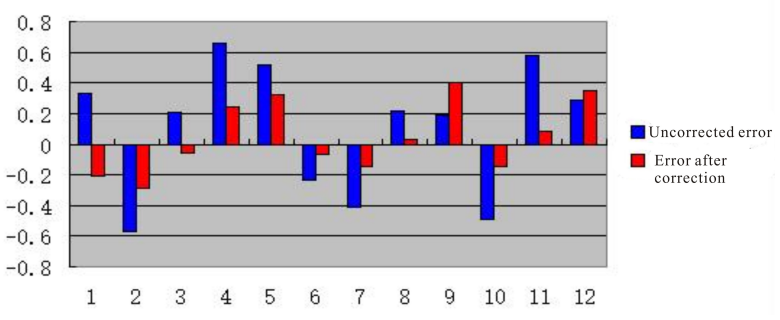

Figure 6 Comparison of polynomial curve fitting accuracy By comparison, the error before the elevation checkpoint correction is $0.343 \mathrm{~m}$, the maximum error is $0.66 \mathrm{~m}$, the correction error is $0.240 \mathrm{~m}$, the maximum error is $0.40 \mathrm{~m}$, and the overall accuracy is increased by 0.300 times. After the elevation checkpoint in the survey area is fitted, the elevation accuracy of the topographic map is improved. The accuracy comparison before and after the elevation of the checkpoint of the survey area is shown in Table 1 below:

Table 1 Accuracy comparison of polynomial curve fitting method before and after elevation fitting

\begin{tabular}{|c|c|c|c|c|}
\hline \multicolumn{2}{|c|}{ Before correction } & \multicolumn{2}{|c|}{ After correction } & \multirow{2}{*}{$\begin{array}{c}\text { Accuracy } \\
\text { improvement } \\
\text { multiple }\end{array}$} \\
\hline $\begin{array}{c}\text { Medium } \\
\text { error }\end{array}$ & $\begin{array}{l}\text { Maximum } \\
\text { error }\end{array}$ & $\begin{array}{c}\text { Medium } \\
\text { error }\end{array}$ & $\begin{array}{l}\text { Maximum } \\
\text { error }\end{array}$ & \\
\hline 0.343 & 0.66 & 0.240 & 0.40 & 0.300 \\
\hline
\end{tabular}

\subsubsection{Elevation Surface Fitting Method}

According to the elevation data of 12 points, the maximum and minimum values of the elevation error of the checkpoint after correction are $0.43 \mathrm{~m}$ and $0.09 \mathrm{~m}$, respectively, and the error in the corrected elevation is also calculated to be $0.286 \mathrm{~m}$. The elevation error distribution before and after the checkpoint correction is shown in Figure 7.
Accuracy comparison of surface fitting method

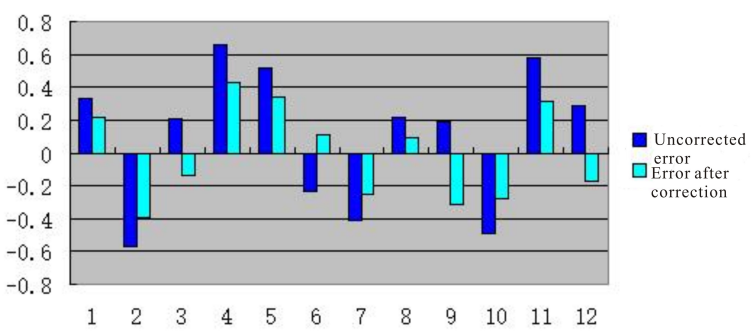

Figure 7 Surface Fitting Method Accuracy Comparison By comparison, the error before the elevation checkpoint correction is $0.343 \mathrm{~m}$, the maximum error is $0.66 \mathrm{~m}$, the correction error is $0.286 \mathrm{~m}$, the maximum error is $0.43 \mathrm{~m}$, and the overall accuracy is improved by 0.166 times. After the elevation checkpoint in the survey area is corrected, the elevation accuracy of the topographic map is improved. The comparison of the accuracy of the checkpoint elevation before and after correction is shown in Table 2.

Table 2 Surface Fitting Method Accuracy Comparison before and after Elevation Correction

\begin{tabular}{|c|c|c|c|c|}
\hline \multicolumn{2}{|c|}{ Before correction } & \multicolumn{2}{|c|}{ After correction } & \multirow{2}{*}{$\begin{array}{c}\text { Accuracy } \\
\text { improvement } \\
\text { multiple }\end{array}$} \\
\hline $\begin{array}{l}\text { Medium } \\
\text { error }\end{array}$ & $\begin{array}{l}\text { Maximum } \\
\text { error }\end{array}$ & $\begin{array}{l}\text { Medium } \\
\text { error }\end{array}$ & $\begin{array}{l}\text { Maximum } \\
\text { error }\end{array}$ & \\
\hline 0.343 & 0.66 & 0.286 & 0.43 & 0.166 \\
\hline
\end{tabular}

\subsubsection{Akima curve fitting method}

According to the elevation data of 12 points, the maximum and minimum values of the elevation error of the checkpoint after correction are $-0.40 \mathrm{~m}$ and $0.11 \mathrm{~m}$, respectively, and the error in the corrected elevation is also calculated to be $0.245 \mathrm{~m}$. The elevation error distribution before and after the checkpoint correction is as shown in Figure 8.

Accuracy comparison of Akima curve fitting method

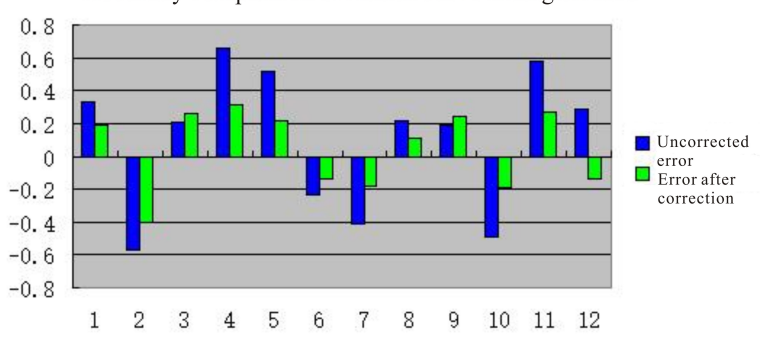

Figure 8 Accuracy comparison of Akima curve fitting method By comparison, the error before the elevation checkpoint correction is $0.343 \mathrm{~m}$, the maximum error is $0.66 \mathrm{~m}$, the correction error is $0.245 \mathrm{~m}$, the maximum error is $-0.40 \mathrm{~m}$, and the overall accuracy is improved by 0.286 times. After the elevation checkpoint in the survey area is corrected, the elevation accuracy of the topographic map is improved. The comparison of the accuracy of the checkpoint elevation before and after correction is shown in Table 3.

Table 3 Accuracy comparison of Akima curve fitting method before and after elevation correction

\begin{tabular}{|c|c|c|c|c|}
\hline \multicolumn{2}{|c|}{ Before correction } & \multicolumn{2}{|c|}{ After correction } & \multirow{2}{*}{$\begin{array}{c}\text { Accuracy } \\
\text { improvement } \\
\text { multiple }\end{array}$} \\
\hline $\begin{array}{c}\text { Medium } \\
\text { error }\end{array}$ & $\begin{array}{l}\text { Maximum } \\
\text { error }\end{array}$ & $\begin{array}{l}\text { Medium } \\
\text { error }\end{array}$ & $\begin{array}{l}\text { Maximum } \\
\text { error }\end{array}$ & \\
\hline 0.343 & 0.66 & 0.245 & -0.40 & 0.286 \\
\hline
\end{tabular}

Through the comparison of the three results of Table 1, Table 2, and Table 3, in the small area and the relatively flat terrain, the three methods of polynomial curve fitting, surface fitting and Akima curve fitting are used to fit the elevation. Both can improve the elevation accuracy level, which not only improves the accuracy requirements, but also improves the work efficiency. By fitting the test area elevation, in the case where 
the fixed check points are selected uniformly, the area is polynomial curve fitting, and the Akim curve fitting accuracy is better than the surface fitting precision. Of course, the selection of fixed checkpoints will also have a certain degree of influence on the accuracy of the fitting results.

\section{CONCLUNSIONS}

This chapter focuses on the overall process of producing digital topographic maps for UAV systems, including internal and external workflows, data inspection, and pre-processing. This paper focuses on the processing of image data using the Southern SouthUAV system, the process of producing DEM and DOM, and the process of making DLG through the Aerospace Vision MapMatrix Digital Photogrammetry System, which converts DLG into a digital topographic map of the Southern CASS mapping software format. In addition, based on the comparative analysis and demonstration of field measurement data, this chapter systematically analyzes the accuracy level that can be achieved, and verifies the operability of the Southern Sky Survey AS1200 UAV aerial photography system in aerial survey digital topographic map. Finally, polynomial curve fitting method, surface fitting method and Akima curve fitting method are used to correct the elevation. The error before correction is 0.343 . The errors in the elevation of the three methods are $0.240,0.286,0.245$, respectively. The accuracy has been improved, and the overall elevation accuracy has been increased by 0.300 times, 0.166 times, and 0.286 times, respectively.

\section{ACKNOWLEDGEMENTS}

This paper is written by young and middle-aged teachers in Guangxi universities.Scientific research basic capacity improvement project support, project number is2019KY1057.

\section{REFERENCES}

Gao, Z.G., Song, Y., Zeng, F.Y., 2015. Experimental analysis of large-scale topographic maps in small area of mini-UAV aerial photography system. Engineering Investigation, 12, pp. 71-75.

Yang, L., 2015. Key Points of 1:500 Large Scale Mapping in Rural Land Survey. China Building Materials Science and Technology, 24(3), pp. 131-133.

Zhang, Y.G., Li, J.F., Xue Z.Y., Wang X.X., 2016. Application of Vehicle Mobile Measurement System in Large Scale Map Measurement and Updating.Beijing Surveying and Mapping, 6, pp. 115-118.

Herwitz, S., Johnson, L., Arvesen, J., et al., 2002. Precision agriculture as a commercial application for solar-powered unmanned aerial vehicles. 1st UAV Conference, pp. 3404.

Herwitz, S. R., Johnson, L. F., Dunagan, S. E., et al., 2004. Imaging from an unmanned aerial vehicle: agricultural surveillance and decision support. Computers and electronics in agriculture, 44(1), pp. 49-61.

Remondino, F., Barazzetti, L., Nex, F., et al., 2011. UAV photogrammetry for mapping and $3 \mathrm{~d}$ modeling-current status and future perspectives. International archives of the photogrammetry, remote sensing and spatial information sciences, 38(1), pp. C22.
Ma, T.C., 2018. Application of UAV Photogrammetry System in Large Scale Mapping. Chengdu University of Technology.

Zheng, H.Q., 2011 .Discussion on Large Scale Mapping Using UAV Aerial Photo. Guangdong Science and Technology, 20(22), pp.183-184.

Shen, J., Tang, W., 2017.Application of UAV aerial photogrammetry in topographic mapping of small farmland water conservancy design.Jiangxi Surveying and Mapping, 03, pp. $22-24+42$.

Lu, X.P., 2014. An Empirical Study on the Accuracy of Lowaltitude Photogrammetry of UAV. Xuzhou: China University of Mining and Technology.

Fu, C.Y., Yu, X.X., Xu, H., Li, N., 2012 .A DEM Production Method Based on High Resolution Airborne SAR. Bulletin of Surveying and Mapping, 4, pp. 50-51.

He, S.L., Xu, J.H., Zhang, S.Y., 2012. Drone image like control discrete point interpolation rules grid DEM data accuracy evaluation. Engineering Survey, 10, pp. 71-76.

Li, M., Liu, D.D., Chen, X., Wang, L., 2012.Comparative Analysis of Several Commonly Made DEM Methods. Jiangxi Surveying and Mapping, 4, pp. 33-34.

Chen, D.P., 2011. Research on Task Planning and Data Processing of Surveying UAV System. Zhengzhou: PLA Information Engineering University.

Zhang, J.Q., Pan, L., Wang, S.G., 2009. Photogrammetry (Second Edition). Wuhan: Wuhan University Press.

Zhang, F.Q., Wang, K.G., 2014. Discussion on the Application and Development of UAV Aerial Survey Technology.Construction Engineering Technology and Design, 34.

Tian C., 2014. Research on the integration of low-altitude digital aerial photography system based on drone. Xi'an: Chang'an University. 